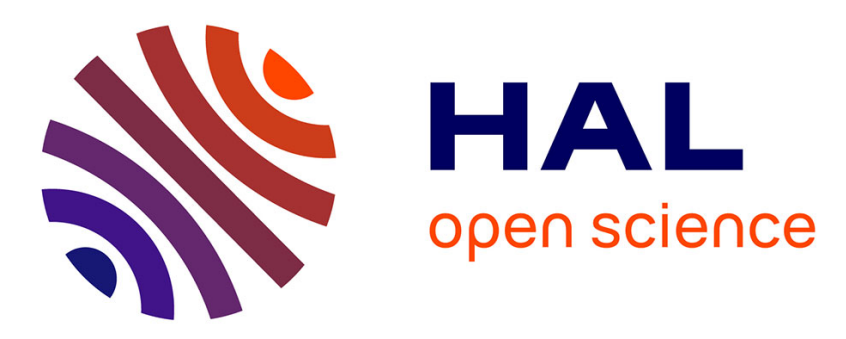

\title{
A class of Petri nets for manufacturing system integration
}

Jean-Marie Proth, Liming Wang, Xiaolan Xie

\section{To cite this version:}

Jean-Marie Proth, Liming Wang, Xiaolan Xie. A class of Petri nets for manufacturing system integration. [Research Report] RR-2055, INRIA. 1993, pp.28. inria-00074617

\section{HAL Id: inria-00074617 https://hal.inria.fr/inria-00074617}

Submitted on 24 May 2006

HAL is a multi-disciplinary open access archive for the deposit and dissemination of scientific research documents, whether they are published or not. The documents may come from teaching and research institutions in France or abroad, or from public or private research centers.
L'archive ouverte pluridisciplinaire HAL, est destinée au dépôt et à la diffusion de documents scientifiques de niveau recherche, publiés ou non, émanant des établissements d'enseignement et de recherche français ou étrangers, des laboratoires publics ou privés. 


\section{A class of Petri Nets for Manufacturing System Integration}

Jean-Marie PROTH

Liming WANG - Xiaolan XIE

$\mathrm{N}^{\circ} 2055$

Octobre 1993

PROGRAMME 5

Traitement du signal, automatique et productique

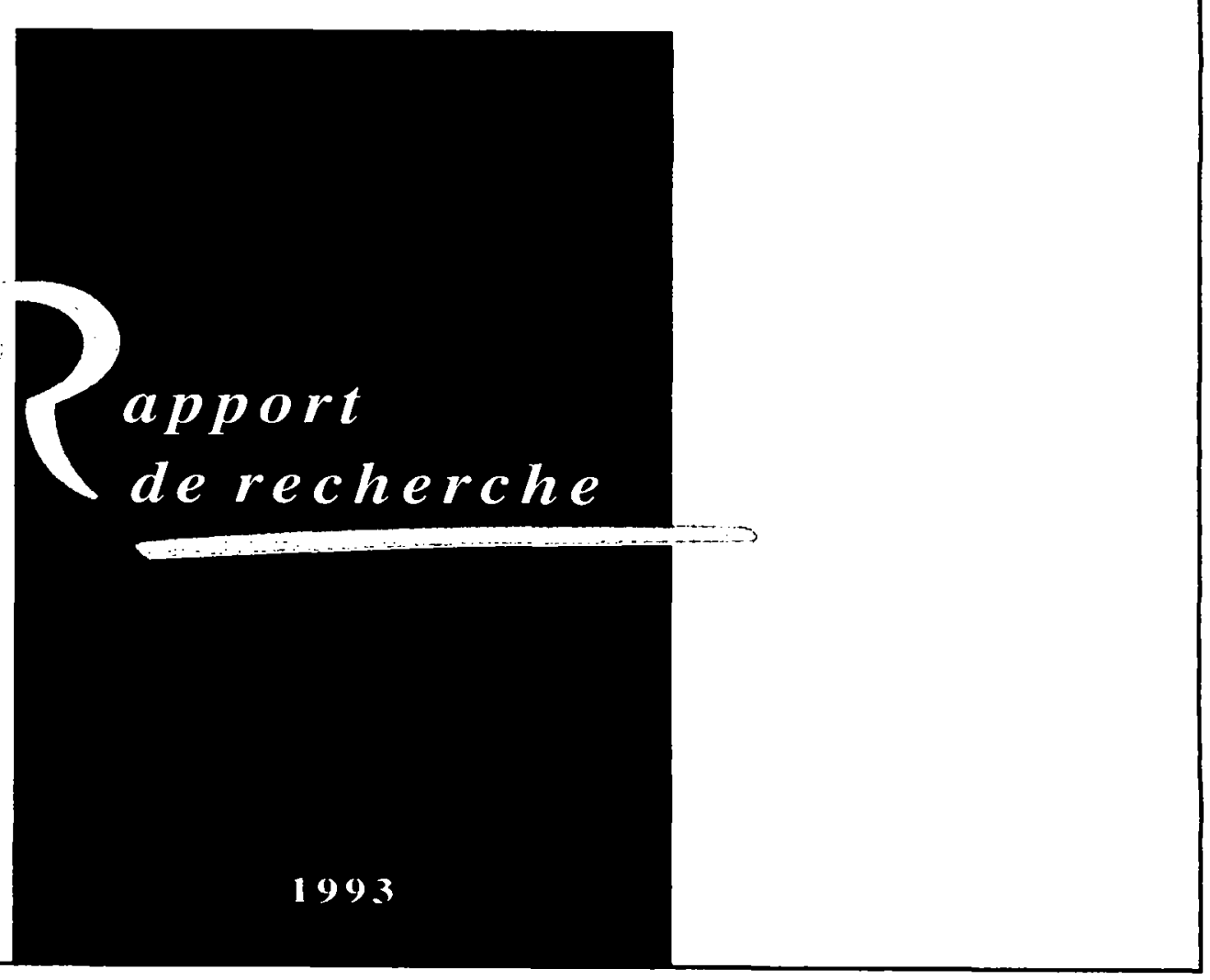




\title{
UNE CLASSE DE RESEAUX DE PETRI POUR L'INTEGRATION DES SYSTEMES DE PRODUCTION
}

\author{
Jean-Marie PROTH, Liming WANG and Xiaolan XIE \\ INRIA, Technopole Metz 2000,4, rue Marconi, 57070 METZ, France
}

\begin{abstract}
Résumé
Ce papier s'intéresse à l'intégration de modèles représentant des modules (ou sous-systèmes) de fabrication. Une méthodology modulaire pour l'intégration étant adoptée, nous définissons une classe de réseaux de Petri applicable à la modélisation d'un large ensemble de systèmes de fabrication. Les réseaux de Petri sont vivant, consistant et réversibles. Nous montrons également que l'intégration de ces modules conserve ces propriétés qualitatives sous des conditions peu restrictives. De plus, le modèle global appartient également à la classe de réseaux de Petri considérée. Les résultats obtenus dans cette communication permettent d'éclairer les problèmes de conception, de gestion et de contrôle des systèmes de production de grande taille.
\end{abstract}

Mots clefs: Systèmes de fabrication, Réseaux de Petri, Intégration 


\title{
A CLASS OF PETRI NETS FOR MANUFACTURING SYSTEM INTEGRATION
}

\author{
Jean-Marie PROTH, Liming WANG and Xiaolan XIE \\ INRIA, Technopole Metz 2000,4, rue Marconi, 57070 METZ, France
}

\begin{abstract}
This paper addresses the problem of integration of Petri nets representing manufacturing systems. A modular modelling methodology being adopted, we define a class of Petri nets that characterizes a large sort of real-world manufacturing systems. These Petri nets are shown to have the properties of liveness, consistency, and reversibility. We also show that the integration of such Petri nets preserves these properties under fairly weak conditions. Moreover the integrated Petri net belongs to the same Petri net class. The results obtained in this paper are expected to shed light on the design, management and control of large scale manufacturing systems where complexity problems are bound to rise and can be dealt with from the point of view of modular modelling and integration.
\end{abstract}

Keywords: Manufacturing systems, Petri nets, Integration 


\section{INTRODUCTION}

A major concern, when modelling a manufacturing system by means of Petri nets, is to check whether the Petri net model has desired qualitative properties such as liveness, boundedness, reversibility, and consistency. These desired properties characterize the dynamic behaviour of a well-designed system. For instance, in the Petri net model of a manufacturing system, the liveness ensures that blocking will never occur, the boundedness guarantees that the number of in-process parts is upper bounded, the reversibility enables the system to come back to its initial state from whatever state it reaches. We also consider consistency, a structural property, which is a necessary condition for the reversibility.

Due to the complexity of manufacturing systems, the size of their Petri net models is usually very large. Classical property checking methods such as coverability tree, invariant analysis and algebraic analysis hardly apply to large-size Petri net models.

There are two approaches for analyzing a large-scale Petri net model. The first one is the reduction of Petri nets while preserving properties. Reduction rules have been proposed $[3,8,9]$. The main disadvantage of this approach lies in the difficulty of finding the reducible sub-Petri nets.

The second approach includes synthesis methods which build the models systematically and progressively such that the desired properties are preserved all along the design process. The basic idea is to adopt a design process which preserves the desired properties in the model instead of checking properties after modelling the system. Two synthesis approaches: top-down approach and bottom-up approach, have been proposed.

The top-down approach begins with an aggregate model of the system which is refined progressively to introduce more and more details. The basic refinement is the substitution of a place or a transition by a so-called well-formed block [14, $15,16]$. Conditions, under which the desired properties are preserved, are given. This approach is well suited to model systems composed of almost independent sub-systems. However, this approach loses its efficiency in case of strongly coupled sub-systems since it is impossible to find a small aggregate model. 
The bottom-up approach (see $[1,5,6,7,11,12]$ ) starts from sub-system models (or module models) and integrate them by merging some places and/or transitions. The disadvantage of the general bottom-up approach lies mainly in the extreme difficulty of checking whether an integration preserves the desired properties.

In this paper, we propose a specific bottom-up approach for modelling manufacturing systems which preserves the qualitative properties of the modules, these qualitative properties being those mentioned above.

This paper is organised as follows. In Section II we introduce some basics of Petri nets. The definition of the class of Petri net modules considered in this paper is presented in Section III and we call them Controllable-Output nets or $\mathrm{CO}$ nets. Some structural and behavioural properties of these $\mathrm{CO}$ modules are studied in Section IV. Section V is devoted to the issue of system integration, from which some important results are obtained. As the $\mathrm{CO}$ modules are inherently unbounded, while boundedness is highly desirable in practice, a separate section, Section VI, is created to tackle this problem. Section VII addresses the identification of $\mathrm{CO}$ modules. In Section VIII, the connection between $\mathrm{CO}$ nets and CFIO-decomposable Petri nets [4] is given from production management point of view. Section IX concludes the paper.

\section{SOME BASIC NOTIONS OF PETRI NETS}

We use ordinary Petri net to model manufacturing systems. Transitions represent operations in the system, places and the connecting arcs specify the logical relationship and resource constraints among operations. A time parameter is attached to each transition to model the duration of the corresponding operation. When time parameter is not represented explicitly, it is called an untimed model, which is what we make use of here since we confine ourselves to qualitative properties of manufacturing systems.

An ordinary Petri net is a 4-tuple $\mathrm{N}=\left(\mathrm{P}, \mathrm{T}, \mathrm{F}, \mathrm{M}_{0}\right)$, where $\mathrm{P}$ and $\mathrm{T}$ are two disjointed sets of nodes called respectively places and transitions. $F \subseteq(P \times T) \cup(T \times P)$ is a set of directed arcs. $M_{0}: P \rightarrow I N$ is the initial marking, where IN is the set of non-negative integers. 
The set of input (respectively output) transitions of a place $p \in P$ is denoted by ${ }^{\circ} p$ (respectively $\mathrm{p}^{\bullet}$ ). Similarly the set of input (respectively output) places of a transition $t \in T$ is denoted by ${ }^{\circ} t$ (respectively $t^{\circ}$ ).

A transition $t \in T$ is said to be enabled at $M_{0}$ if for all $p \in \epsilon^{\bullet} t, M_{0}(p) \geq 1$. A transition may fire if it is enabled. The firing of a transition $t$ at marking $M$ removes one token from each of its input places and put one token to each of its output places, thus leads to a new marking, say $\mathrm{M}^{\prime}$. This process is denoted by $M\left[t>M^{\prime}\right.$. If $M^{\prime}$ is not explicitly mentioned, the process is denoted by $M[t>$, which means that $t$ is firable at $M$. These notations are also extended to sequences of firings, i.e., $M\left[\sigma>M^{\prime}\right.$, where $\sigma$ is a sequence of transitions that brings $M$ to $M^{\prime}$, and $\mathrm{M}\left[\sigma>\right.$, if $\mathrm{M}^{\prime}$ is not explicitly mentioned. The set of all markings reachable from $M_{0}$ is denoted by $R\left(M_{0}\right)$.

The structure of a Petri net is represented by its incidence matrix $C=\left[c_{i j}\right], i=1, \ldots,|P|, j=1, \ldots,|T|$, where

$$
c_{i j}= \begin{cases}1, & \text { if }\left(t_{j}, p_{i}\right) \in F \\ -1 & \text { if }\left(p_{i}, t_{j}\right) \in F \\ 0 & \text { otherwise }\end{cases}
$$

$|\mathrm{A}|$ is the number of elements in the set $\mathrm{A}$.

A $|T| \times 1$ non-negative integer vector $y$ such that $C y=0$ and $y \neq 0$ is called a $T$ invariant of the net. A T-invariant $y$ is called minimal if there does not exist a $T$-invariant $x$ such that $x \leq y$. The set $\|y\|=\{t \mid y[t]>0\}$ of transitions is called the support of T-invariant $y$, where $y[t]$ is the entry corresponding to transition $t$. $\|y\|$ is said to be minimal if it does not contain another $T$-invariant support. The minimal T-invariant y corresponding to the minimal support $\|\mathrm{y}\|$ is called a minimal support T-invariant. A Petri net is said to be consistent if and only if there exists a positive T-invariant for this net. A Petri net is reversible if, for each marking $M$ in $R\left(M_{0}\right), M_{0}$ is reachable from $M$, i.e., $M_{0} \in R(M)$. 


\section{A CLASS OF MANUFACTURING MODULE}

DEFINITION III-1: (CO net module-Controllable-Output net module) A Petri net $G=\left(P \cup R, T, F, M_{0}\right)$, where $P$ and $R$ are two disjointed sets of process places and resource places, is called a controllable-output net module (CO net module) if the following conditions hold:

H1. $(\mathrm{t}, \mathrm{r}) \in \mathrm{F} \Leftrightarrow(\mathrm{r}, \mathrm{t}) \in \mathrm{F}, \quad \forall \mathrm{t} \in \mathrm{T}, \quad \forall \mathrm{r} \in \mathrm{R}$

H2. $M_{0}(r) \geq 1, \forall r \in R$

H3. The subnet $G^{\prime}=\left(P, T, F^{\prime}, M_{0}^{\prime}\right)$, where $F^{\prime}$ is the restriction of $F$ on $(P \times T) \cup(T \times P)$, is an acyclic graph without isolated nodes. $M^{\prime}$ is the restriction of $\mathrm{M}_{0}$ on $\mathrm{P}$.

H4. The extremity nodes of $G$ are transitions. Let $T_{\text {in }}$ and $T_{\text {out }}$ be respectively the set of input transitions and the set of output transitions, i.e.

$$
T_{\text {in }}=\left\{\left.t\right|^{\bullet} t=\varnothing, t \in T\right\}, T_{\text {out }}=\left\{t \mid t^{\bullet}=\varnothing, t \in T\right\} .
$$

Note that due to $\mathrm{H} 1$ and $\mathrm{H} 3, \mathrm{~T}_{\mathrm{in}}$ and $\mathrm{T}_{\text {out }}$ are subsets of transitions of $\mathrm{G}^{\prime}$.

H5. The net $G^{\prime}$ is covered by a set of $T$-invariants each of which is related to a unique output transition, i.e., for all $t \in T_{\text {out }}, \exists y_{t} \in I^{|T|}$, such that

$$
C y_{t}=0,\left\|y_{t}\right\| \cap T_{\text {out }}=\{t\} \text {, and } \underset{t \in T_{\text {out }}}{U}\left\|y_{t}\right\|=T
$$

Remark III-1: $\mathrm{H} 1$ and $\mathrm{H} 2$ together imply that resource places $\mathrm{r} \in \mathrm{R}$ are implicit places that are, informally speaking, those places whose markings are always sufficient for the firing of their output transitions. According to [10], we know that removing the implicit places does not change the behaviour of the net. As a result, we only need to consider the net $\mathrm{G}^{\prime}$.

Remark III-2: Hypothesis H3 and H4 imply that any place is an internal node, i.e., it is neither a sink place nor a source place. Furthermore, the set of input transitions $\mathrm{T}_{\mathrm{in}}$ and the set of output transitions $\mathrm{T}_{\text {out }}$ are disjointed, i.e., $\mathrm{T}_{\text {in }} \cap \mathrm{T}_{\text {out }}=\varnothing$.

Remark III-3: Hypothesis H5 implies that the net $\mathrm{G}^{\prime}$ is consistent. But a consistent net does not display hypothesis $\mathrm{H} 5$ as shown in Figure 1(a). It is the requirement of $\mathrm{H} 5$ that motivates us to term this class of Petri net modules as 
controllable-output net modules, since $\mathrm{H} 5$ requires that each output transition of the module is related to at least one T-invariant the support of which does not contain any other output transitions of the module. That is to say, the firing of each output transition can be totally independent of the firings of other output transitions. Thus the output of the systems represented by $\mathrm{CO}$ nets can be controlled with great flexibility.

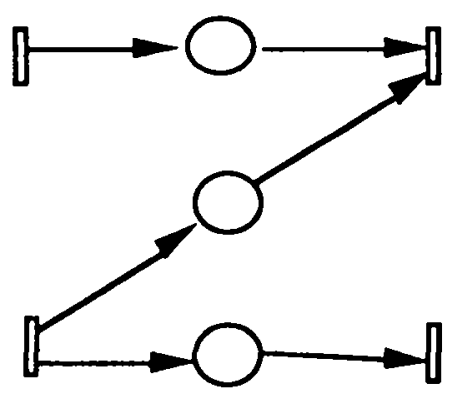

(a)

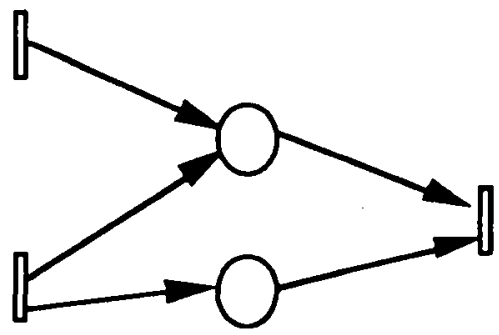

(b)

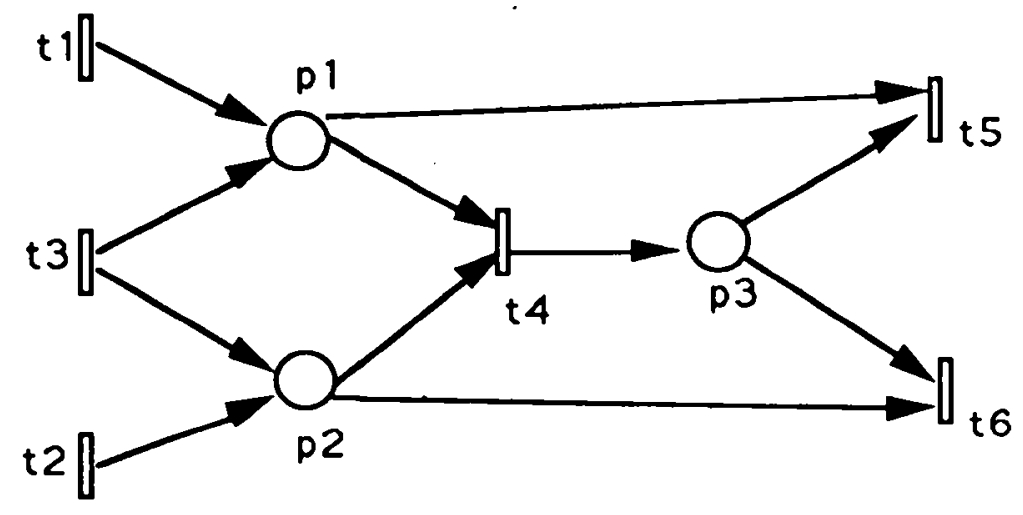

(c)

Fig. 1: CO nets (c) and non-CO nets (a),(b)

Figure 1 gives three Petri nets in which (c) is a $\mathrm{CO}$ net, while (a), (b) are not since the net (b) is not consistent, and (a) has only one T-invariant the support of which contains both output transitions, thus H5 is violated. Note that for simplicity resource places and connecting arcs are not shown in these Petri net graphs. The reason that (c) is a $\mathrm{CO}$ net is that there exist two T-invariants $y_{t_{5}}=\left[\begin{array}{llllll}1 & 0 & 1 & 1 & 1 & 0\end{array}\right]$ and $y_{t_{6}}=\left[\begin{array}{llllll}0 & 1 & 1 & 1 & 0 & 1\end{array}\right]$, each concerning exactly one output transition and the union of their supports covers all the transitions of the net. 


\section{PROPERTIES OF CO NETS}

In this section we investigate some structural and behavioural properties of $\mathrm{CO}$ nets. Specifically we are interested in consistency, liveness, boundedness, reversibility, which have been justified in the introduction.

As a result of remark III-1, we only need to consider CO nets $G$ without any resource places, i.e., $R=\varnothing$. In this case $G$ ' is identical to $G$.

RESULT IV-1: A CO net $\mathrm{G}$ is consistent, if $\mathrm{R}=\varnothing$.

PROOF: It is obvious from assumption H5.

Q.E.D

RESULT IV-2: A CO net $G$ is live, if $R=\varnothing$, no matter what the initial marking is.

PROOF: Since $G$ is an acyclic graph, we can relabel the transitions as $t_{1}, t_{2}$, $\ldots, t_{n}$ ( $n$ is the number of transitions in $G$ ) such that $\forall t_{i} \in T$, no path exists from $t_{i+k}$ to $t_{i}(k=1,2, \ldots, n-i)$, and the first transition(s), say $t_{1}, t_{2}, \ldots, t_{r}(1 \leq r<n)$ are input transition(s).

In the following, we show by induction that

$$
\forall t_{i} \in T, \exists \sigma_{i} \in\left\{t_{1}, t_{2}, \ldots, t_{i-1}\right\}^{*} \text {, such that } M\left[\sigma_{i} \circ t_{i}>, \forall M \in(I N)^{|P|}\right.
$$

where $A^{*}$ is the set of all finite sequences (including empty sequence, denoted by $\lambda$ ) formed by the elements in the finite set $A$, and $\sigma \circ t$ represents the concatenation of a sequence $\sigma$ with an element $t$.

(a) First, induction assumption holds for any input transition $t_{i} \in\left\{t_{1}, t_{2}, \ldots, t_{r}\right\}$, i.e., $\exists \sigma_{0}=\lambda$ such that $M\left[\sigma_{0} \circ t_{i}>, \forall M \in(I N)^{|P|}\right.$.

(b) We then assume that claim (4-1) holds for any $\mathrm{t}_{\mathrm{i}}$ such that $\mathrm{i} \leq \mathrm{k}<|\mathrm{T}|$. Consider transition $t_{k+1}$, all the transitions which immediately precede $t_{k+1}$, i.e., the transitions belonging to ${ }^{\bullet}\left(t_{k+1}\right)$, belong to $\left\{t_{1}, t_{2, \ldots,} t_{k}\right\}$. This is the consequence of the way transitions have been re-labelled. 
Thus if all the transitions of ${ }^{\bullet}\left({ }^{\bullet} t_{k+1}\right)$ have been fired, it is possible to fire $t_{k+1}$. Furthermore, according to the induction assumption, $\forall \mathrm{t}_{\mathrm{j}} \in^{\bullet}\left(\mathrm{t}_{\mathrm{k}+1}\right)$, $\exists \sigma_{j} \in\left\{t_{1}, t_{2}, \ldots, t_{j-1}\right\}^{*}$ such that $M\left[\sigma_{j} \circ t_{j}>, \forall M \in(I N)^{|P|}\right.$. As a consequence, $\mathrm{M}\left[\sigma_{\mathrm{k}+1} \circ \mathrm{t}_{\mathrm{k}+1}>, \forall M \in(\mathrm{IN})^{|\mathrm{P}|} \quad\right.$ holds $\quad$ if $\quad \sigma_{\mathrm{k}+1}=\underset{\mathrm{s} \in \mathrm{E}_{\mathrm{k}+1}}{\mathrm{O}}\left(\sigma_{\mathrm{s}} \circ \mathrm{t}_{\mathrm{s}}\right), \quad$ where $E_{k+1}=\left\{j / t_{j} \in^{\bullet}\left({ }^{\bullet} t_{k+1}\right)\right\}$, and $O$ stands for successive concatenation.

We notice that another simple proof may be proposed. Since the net G does not contain any siphon, it is live for any initial marking $\mathrm{M}_{0}$ according to [2]. As can be noticed, this property holds for any acyclic Petri net without source place(s).

RESULT IV-3: A CO net G is not bounded, if $R=\varnothing$.

PROOF: This result is due to the existence of input transitions.

Q.E.D.

Furthermore since CO net is consistent, and the subnet structure is acyclic, we claim that

RESULT IV-4: An CO net $G$ is reversible, if $R=\varnothing$, whatever the initial marking is.

PROOF: For any $M \in R\left(M_{0}\right)$, the proof is as follows;

(a) $\exists \sigma \in T^{*}$ such that $M_{0}[\sigma>M$.

(b) Since $G$ is consistent, then there exists positive $|T| \times 1$ vector $x$, such that $\mathrm{Cx}=0$.

(c) From (a) and (b), we have that there exists a positive integer $k$, such that $k x-\bar{\sigma} \geq 0$, and $k x-\bar{\sigma} \neq 0$ where $\bar{\sigma}$ is the firing count vector of $\sigma$.

which yields

(d) $\mathrm{M}_{0}=\mathrm{M}+\mathrm{C}(\mathrm{kx}-\bar{\sigma})$

(e) From Theorem 16 in [10], (d) and (c) implies that $M_{0}$ is reachable from $M$. 
In view of remark III-1, the above results can be extended to the general case, i.e., $R \neq \varnothing$.

RESULT IV-5: For a $C O$ net $G$ with any initial marking $M_{0}(p)$ of process places, i.e., $p \in P$,
(a) $\mathrm{G}$ is consistent.
(b) $\mathrm{G}$ is live.
(c) $\mathrm{G}$ is unbounded.
(d) $G$ is reversible.

Remark IV-1: We would like to emphasize that in the proof of the above properties of $\mathrm{CO}$ nets, only some assumptions are used. For liveness, only acyclicity is used; for unboundedness, only the input-output characteristic of CO net is used, while reversibility is the consequence of acyclicity and consistency.

\section{SYSTEM INTEGRATION}

In Section IV we studied some properties of $\mathrm{CO}$ nets, and obtained the result that a $\mathrm{CO}$ net is consistent, live, and reversible, though not bounded. Usually several manufacturing modules are linked together to accomplish a certain manufacturing function. Knowing that each module has the qualitative properties introduced above, the problem is to select an integration process which preserves these properties, i.e., which guarantees that the integrated system still has these qualitative properties. We consider the integration process in which all the $\mathrm{CO}$ net modules are linked together through a set $\mathrm{Q}$ of places, called interface places.

\section{DEFINITION V-1: (integrated system )}

Consider a set of modules $G_{1}, G_{2}, \ldots, G_{n}$, where $G_{i}=\left(P_{i} \cup R_{i}, T_{i}, F_{i}, M_{0 i}\right)$ with input transitions $\mathrm{T}_{\mathrm{in}}^{\mathrm{i}}$ and output transitions $\mathrm{T}_{\text {out }}^{\mathrm{i}}$. A integrated system $\mathrm{G}$ is a Petri net resulting from the integration of modules $G_{1}, G_{2}, \ldots, G_{n}$ via the set of interface places $Q$ and inter-module arcs $\Gamma$

$$
G=\left(\bigcup_{i=1}^{n} P_{i} \cup R_{i} \cup Q, \bigcup_{i=1}^{n} T_{i}, \bigcup_{i=1}^{n} F_{i} \cup \Gamma\right)
$$

where

$$
\Gamma=\left(\bigcup_{i=1}^{n} T_{\text {out }}^{i} \times Q\right) \cup\left(Q \times \bigcup_{i=1}^{n} T_{i n}^{i}\right)
$$


We further make the following assumptions about interfaces:

H6. Each interface place is neither a source place nor a sink place.

H7. Each output transition of any module is connected to at most one interface place, i.e.,

$$
\left|t^{\bullet}\right| \leq 1 \quad \forall t \in \bigcup_{i=1}^{n} T_{\text {out }}^{i}
$$

A typical system satisfying $\mathrm{H} 6$ and $\mathrm{H} 7$ is shown in Figure 2.

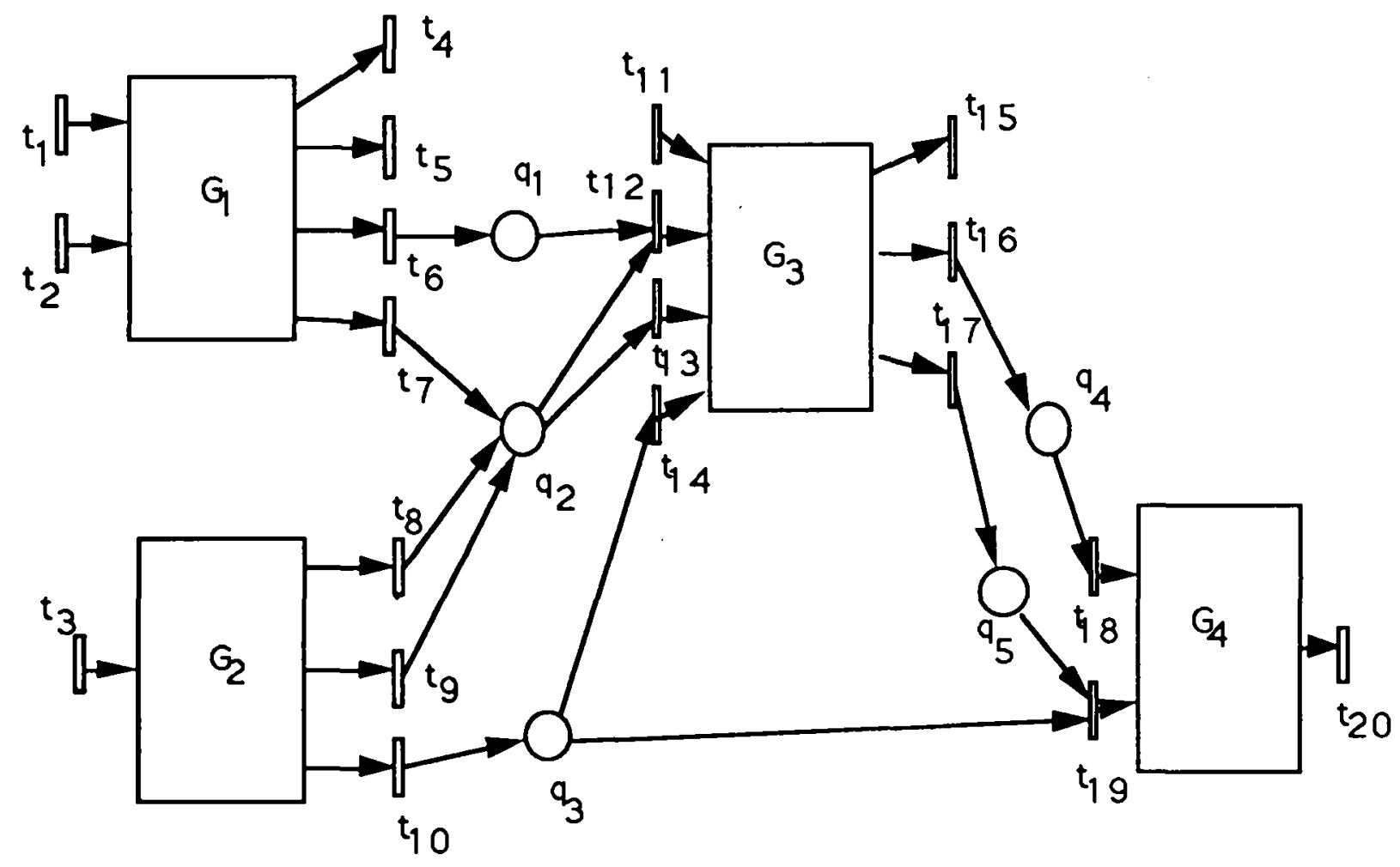

Fig. 2: A typical structure of system integration

\section{DEFINITION V-2: ( system contraction )}

System contraction is the process of transforming an integrated system into a directed graph ( called contracted graph) by contracting each module of the system as a node, viewing each place $q \in Q$ and each transition $t \in\left\{s / s \in T_{i n}^{\mathrm{i}} \cup T_{\text {out }}^{\mathrm{i}}\right.$, for all mod ules $G_{i}$ in the system $\}$ as a node, and preserving the inter-module arcs.

The contracted graph of the system shown in Fig. 2 (a) is drawn in Figure 3. 
DEFINITION V-3: ( acyclic system )

An acyclic system formed by modules $G_{1}, G_{2}, \ldots, G_{n}$ via the set of interface places $Q$ and inter-module arcs $\Gamma$ is a system

$$
G=\left(\bigcup_{i=1}^{n} P_{i} \cup R_{i} \cup Q, \bigcup_{i=1}^{n} T_{i}, \bigcup_{i=1}^{n} F_{i} \cup \Gamma\right)
$$

whose contracted graph is acyclic.

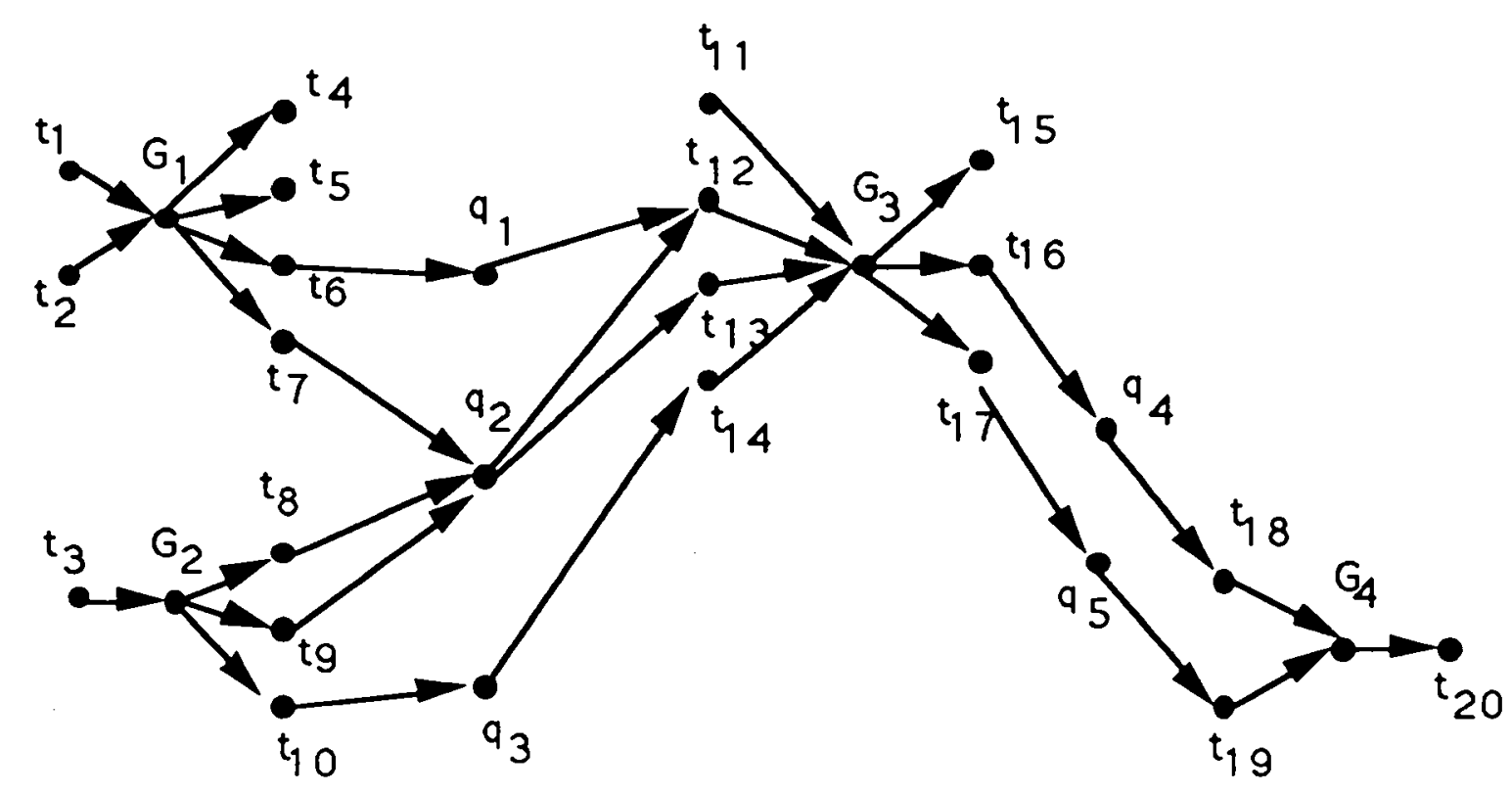

Fig. 3: A contracted graph

\section{RESULT V-1:}

An acyclic system $\mathrm{G}$ satisfying $\mathrm{H} 6$ and $\mathrm{H} 7$ is consistent.

\section{PROOF:}

From the definition of acyclic system, it is always possible to relabel the modules as $G_{1}, G_{2}, \ldots, G_{n}$, such that there are no directed paths from $G_{j}$ to $G_{i}$ if $\mathrm{j}>\mathrm{i}, \mathrm{i}, \mathrm{j}=1,2, \ldots, \mathrm{n}$.

For each module $G_{i}$, let $\left\{y_{t} / \forall t \in T_{\text {out }}^{i}\right\}$ be a set of $T$-invariants satisfying assumption H5, i.e., 


$$
\underset{t \in T_{\text {out }}^{\mathrm{i}}}{\bigcup}\left\|\mathrm{y}_{\mathrm{t}}\right\|=\mathrm{T}^{\mathrm{i}}
$$

and

$$
\mathrm{y}_{\mathrm{t}}[\mathrm{t}]>0 \text { and } \mathrm{y}_{\mathrm{t}}[\mathrm{s}]=0 \quad \forall \mathrm{s} \in \mathrm{T}_{\text {out }}^{\mathrm{i}}-\{\mathrm{t}\}
$$

The central idea of the proof consists in constructing a T-invariant $Y>0$ of the integrated model. Clearly $\mathrm{Y}$ can be written in the following form $\left[\mathrm{Y}_{1}^{\mathrm{T}} \mathrm{Y}_{2}^{\mathrm{T}} \ldots \mathrm{Y}_{\mathrm{n}}^{\mathrm{T}}\right]^{\mathrm{T}}$ where $\mathrm{Y}_{\mathrm{i}}$ is a vector whose components concern the transitions of $\mathrm{G}_{\mathrm{i}}$.

Two conditions are to be considered. First, $\mathrm{Y}_{\mathrm{i}}>0$ is also a T-invariant of $\mathrm{G}_{\mathrm{i}}$. Second, the T-invariant $\mathrm{Y}$ should satisfy the balance equations for all interface places Q, i.e.,

$$
\sum_{s \in \in^{\bullet} \mathbf{q}} \mathrm{Y}[\mathrm{s}]=\sum_{s \in \mathrm{q}^{\bullet}} \mathrm{Y}[s] \quad \text { for all } \mathrm{q} \in \mathrm{Q}
$$

In the following we construct $\mathrm{Y}$ in a backward fashion. Computing firstly $\mathrm{Yn}$, we then construct $Y_{n-1}, Y_{n-2}, \ldots, Y_{1}$ consecutively.

When computing $Y_{i}$ of module $G_{i}$, we first compute the components corresponding to its output transitions. For this purpose, consider a positive number $x_{t}$ for all $t \in T_{\text {out }}^{i}(i=1,2, \ldots, n)$ defined as follows,

$$
x_{t}= \begin{cases}1, & \text { if } t^{\bullet}=\varnothing \\ \sum_{s \in q^{\bullet}} Y[s] & \text { if } t^{\bullet}=q\end{cases}
$$

where $q$ is the unique output place of $t$,

From the definition of $G_{i}$, each transition $s \in q^{\bullet}$ is an input transition of some of the modules $G_{i+1}, \ldots, G_{n}$, which implies that $Y[s]$ is known. Thus $x_{t}$ is uniquely determined.

The vector $Y_{i}$ is thus defined as follows: 


$$
Y_{i}=\sum_{t \in T_{\text {out }}^{i}} \frac{x_{t}}{y_{t}[t]} y_{t}
$$

It is easily verified that $Y_{i}[t]=x_{t}$ for all $t \in T_{\text {out }}^{i}$ due to relation (5-2).

Since all $x_{t}$ 's are positive real numbers, relation (5-1) implies that $Y_{i}$ is also a $T$-invariant of $G_{i}$ and $Y_{i}>0$.

We still need to prove that the vector $Y$ satisfies the flow balance equation for every interface place $q \in Q$, i.e., equation (5-3). This is done as follows

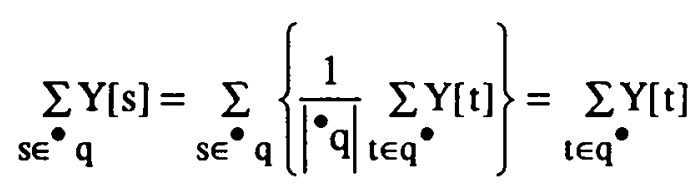

where the first equality is true since $q$ is the unique output place of all $t \in^{\bullet} q$.

Q.E.D.

Furthermore we have the following result:

RESULT V-2: An acyclic system $\mathrm{G}$ satisfying $\mathrm{H} 6$ and $\mathrm{H} 7$ is live, reversible, but not bounded.

PROOF: Since G is an acyclic net without source and sink places and since it is consistent, this result follows from remark IV-1 and result V-1.

Q.E.D

From results V-1 and V-2, we notice that the integration preserves both structural and behavioural properties of modules. This is not a surprise as we can prove that the integrated model is itself a controllable-output net module i.e., a $\mathrm{CO}$ net.

\section{RESULT V-3:}

The integrated system $\mathrm{G}$ satisfies assumptions $\mathrm{H} 1-\mathrm{H} 5$.

\section{PROOF:}

First, we explicitly write $G$ in the following form: 


$$
G=\left(P \cup R, T, F, M_{0}\right) \text {, with } P=\bigcup_{i=1}^{n} P_{i} \cup Q, R=\bigcup_{i=1}^{n} R_{i}, T=\bigcup_{i=1}^{n} T_{i}, F=\bigcup_{i=1}^{n} F_{i} \cup \Gamma \text {. }
$$

Clearly, G satisfies assumptions $\mathrm{H} 1-\mathrm{H} 4$. We now prove that assumption $\mathrm{H} 5$ also holds.

For this purpose, let $T_{\text {in }}$ and $T_{\text {out }}$ be respectively the set of input transitions and the set of output transitions of $G$. Obviously, we have $T_{\text {in }} \subseteq \bigcup_{i=1}^{n} T_{i n}^{i}, T_{\text {out }} \subseteq \bigcup_{i=1}^{n} T_{\text {out }}^{i}$. For each output transition $\tau \in T_{\text {out }}$, let us construct a $T$-invariant $Y_{\tau}$ in a similar way as the one used for constructing $\mathrm{Y}$ in the proof of result $\mathrm{V}-1$, i.e.,

$$
\begin{aligned}
Y_{\tau, i}=\sum_{t \in T_{\text {out }}^{i}} \frac{x_{\tau, t}}{y_{t}[t]} y_{t}, \text { with } \\
x_{\tau, t}= \begin{cases}1, & \text { if } t=\tau \\
0, & \text { if } t \in T_{\text {out }}-\{\tau\} \\
\frac{1}{|\bullet q|} \sum_{s \in q^{\bullet}} Y_{\tau}[s], & \text { if } t^{\bullet}=q\end{cases}
\end{aligned}
$$

where $Y_{\tau, i}, x_{\tau, t}, y_{t}$ are respectively similar notations as $Y_{i}, x_{t}, y_{t}$ defined in the proof of result $V-1$, and $q$ is the unique output place of $t$.

As in the proof of result $\mathrm{V}-1$, it can be shown that $\mathrm{Y}_{\tau}$ is a T-invariant. Now let us prove by induction that the following relation holds:

$$
Y=\sum_{\tau \in T_{\text {out }}} Y_{\tau}
$$

or equivalently:

$$
Y_{i}=\sum_{\tau \in T_{\text {out }}} Y_{\tau, i}, \quad \text { for } \mathrm{i}=1, \ldots, \mathrm{n}
$$

where $\mathrm{Y}$ is the T-invariant of $\mathrm{G}$ used in the proof of result $\mathrm{V}-1$.

It is easy to show that (5-5) holds for $i=n$. Assume that it holds for all $i=k+1$, $k+2, \ldots, n$. Consider then the module $G_{k}$. From the definition of $x_{\tau, t}$ and $x_{t}$, we have 


$$
\sum_{\tau \in \mathrm{T}_{\text {out }}} \mathbf{x}_{\tau, \mathrm{t}}=\mathrm{x}_{\mathrm{t}}=1, \quad \forall \mathrm{t} \in \mathrm{T}_{\text {out }} \cap \mathrm{T}_{\text {out }}^{\mathrm{k}}
$$

Furthermore, by induction assumption, for all $t \in T_{\text {out }}^{k}$, such that $t^{\bullet}=q$,

$$
\sum_{\tau \in \mathrm{T}_{\text {out }}} \mathrm{x}_{\tau, \mathrm{t}}=\sum_{\tau \in \mathrm{T}_{\text {out }}} \frac{\sum_{s \in \mathrm{q}^{\bullet}} \mathrm{Y}_{\tau}[\mathrm{s}]}{|\cdot \mathrm{q}|}=\frac{\sum_{s \in \mathrm{q}^{\bullet}} \mathrm{Y}[\mathrm{s}]}{|\bullet \mathrm{q}|}=\mathrm{x}_{\mathrm{t}}
$$

since all transitions $s \in q^{\bullet}$ belong to modules $G_{k+1}, G_{k+2}, \ldots, G_{n}$ as a result of acyclicity. The above two relations imply that $\sum_{\tau \in T_{\text {out }}} x_{\tau_{t, t}}=x_{t}$, for all $t \in T_{\text {out }}^{k}$, which yields that

$$
\sum_{\tau \in T_{\text {out }}} Y_{\tau, k}=\sum_{\tau \in T_{\text {out }}} \sum_{t \in T_{\text {out }}^{k}} \frac{x_{\tau, t}}{y_{t}[t]} y_{t}=\sum_{t \in T_{\text {out }}^{k}} \frac{x_{t}}{y_{t}[t]} y_{t}=Y_{k}
$$

Equation (5-4) is then proved. Since $\mathrm{Y}$ is a $\mathrm{T}$-invariant and $\mathrm{Y}>0$, we conclude

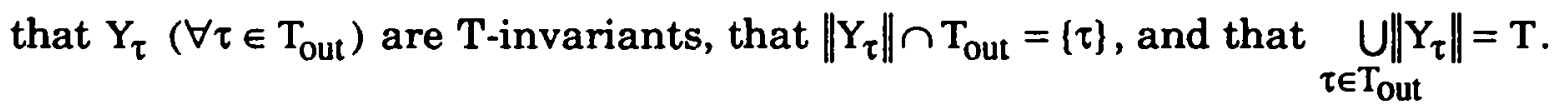

Q.E.D

\section{K-FEASIBLE SEQUENCES}

In Section IV, we have seen that a CO net is consistent, and that it is also live and reversible no matter what the initial marking of the process places is. Unfortunately it is not structurally bounded, while in practice boundedness is highly desirable. This leads us to investigate the issue of boundedness control. Let $G=\left(N, M_{0}\right)$ be a $C O$ net, and let $L(G)=\left\{\sigma \mid M_{0}[\sigma>M\}\right.$. We first define the notion of $k$-feasible sequences and then give a necessary and sufficient condition for the existence of $\mathbf{k}$-feasible sequences for $\mathrm{G}$.

We emphasize that the result is also applicable to any ordinary Petri nets. 
DEFINITION VI-1: ( $k$-feasible sequence )

A sequence $\sigma=\tau_{1} \tau_{2} \ldots \tau_{n}$ of $L(G)$ is called a $k$-feasible sequence if $M_{i}(p) \leq k, \forall p \in P$ for any marking $M_{i}$ obtained by the firing of a sub-sequence $\tau_{1} \tau_{2} \ldots \tau_{i}$ of $\sigma$, for $0 \leq \mathrm{i} \leq \mathrm{n}$.

DEFINITION VI-2: A subset $L_{c}(G)$ of $L(G)$ is prefix-closed if for any sequence $\sigma=\mu \circ \omega \in \mathrm{L}_{c}(\mathrm{G}), \mu \in \mathrm{L}_{\mathrm{c}}(\mathrm{G})$.

RESULT VI-1: For a CO net $G$, a prefix-closed subset $L_{c}(G)$ of $L(G)$ is a set of kfeasible sequences for some finite $k$ if and only if there exists finite $k^{\prime}>0$ such that for any $\sigma \in \mathrm{L}_{\mathrm{c}}(\mathrm{G})$,

$$
\max _{t \in T}\left|\bar{\sigma}[t]-y_{\sigma}[t]\right| \leq k^{\prime}, \text { for at least one T-invariant } y_{\sigma} \text {. }
$$

\section{PROOF:}

a. Sufficient condition:

Since $L_{c}(G)$ is prefix-closed, it is enough to show that any marking reachable via an element of $L_{c}(G)$ is bounded by some finite $k$. Consider a marking $M$ reachable from $M_{0}$ via a sequence $\sigma \in L_{c}(G)$. We have

$$
M=M_{0}+C \bar{\sigma}
$$

where $\bar{\sigma}$ is the firing count vector of $\sigma$.

As $\sigma \in \mathrm{L}_{\mathrm{c}}(\mathrm{G})$, there exists a $\mathrm{T}$-invariant $\mathrm{y}_{\sigma}$ such that

$$
\max _{t \in \mathrm{T}}\left|\bar{\sigma}[\mathrm{t}]-\mathrm{y}_{\sigma}[\mathrm{t}]\right| \leq \mathrm{k}^{\prime}
$$

Combining the above relations, we have

$$
\mathrm{M}(\mathrm{p})=\mathrm{M}_{0}(\mathrm{p})+\mathrm{C}_{\mathrm{p}}\left(\bar{\sigma}-\mathrm{y}_{\sigma}\right) \leq \mathrm{M}_{0}(\mathrm{p})+\left(\cdot \mathrm{p}|+| \mathrm{p}^{\bullet} \mid\right) \mathrm{k}^{\prime}, \quad \forall \mathrm{p} \in \mathrm{P}
$$

where $C_{p}$ is the row vector of incidence matrix $C$ corresponding to $p$. This implies:

$$
\mathrm{M}(\mathrm{p}) \leq \mathrm{k}, \forall \mathrm{p} \in \mathrm{P}, \forall \mathrm{M}_{0}\left[\sigma>\mathrm{M} \text { with } \sigma \in \mathrm{L}_{\mathrm{c}}(\mathrm{G})\right.
$$

where

$$
\mathrm{k}=\max _{\mathrm{p} \in \mathrm{P}}\left\{\mathrm{M}_{0}(\mathrm{p})+\left(\left.\right|^{\bullet} \mathrm{p}|+| \mathrm{p}^{\bullet} \mid\right) \mathrm{k}^{\prime}\right\}
$$

b. Necessary condition:

For all $\sigma \in \mathrm{L}_{\mathrm{c}}(\mathrm{G})$, let $\sigma=\tau_{1} \tau_{2} \ldots \tau_{\mathrm{n}} \quad\left(\tau_{\mathrm{i}} \in \mathrm{T}\right), \quad \sigma_{\mathrm{i}}=\tau_{1} \ldots \tau_{\mathrm{i}}, \quad \mathrm{s}_{\mathrm{i}}=\tau_{\mathrm{i}+1} \ldots \tau_{\mathrm{n}}$, $\mathbf{M}_{0}\left[\sigma_{\mathrm{i}}>\mathrm{M}_{\mathrm{i}}\right.$. Obviously, $\sigma=\sigma_{\mathrm{i}} \mathrm{s}_{\mathrm{i}}$, and $\sigma_{\mathrm{i}} \in \mathrm{L}_{\mathrm{c}}(\mathrm{G})$. If $\mathrm{M}_{0}, \ldots, \mathrm{M}_{\mathrm{n}}$ are distinct, then 


$$
|\sigma|=\mathrm{n} \leq \operatorname{card}\left(\mathrm{R}_{\mathrm{c}}\left(\mathrm{M}_{0}\right)\right)=(\mathrm{k}+1)^{|\mathrm{P}|}
$$

where $R_{c}\left(M_{0}\right)$ is the set of markings reachable from $M_{0}$ via the sequences of transitions of $\mathrm{L}_{\mathrm{c}}(\mathrm{G})$.

Otherwise, assume that $M_{i}=M_{j}(i<j)$, clearly the counting vector of $r^{(1)}=\tau_{i+1} \ldots \tau_{j}$ is a T-invariant, and $\sigma^{(1)}=\sigma_{\mathrm{i}} \mathrm{s}_{\mathrm{j}} \in \mathrm{L}_{\mathrm{c}}(\mathrm{G})$. Moreover,

$$
\bar{\sigma}=\overline{\sigma^{(1)}}+\overline{r^{(1)}}
$$

Repeating the above reasoning, we obtain a set of sequences $r^{(1)}, \ldots, r^{(h)}$ whose counting vectors are T-invariants, and a set of sequences $\sigma^{(1)}, \ldots, \sigma^{(h)} \in L_{c}(G)$, such that

$$
\overline{\sigma^{(j)}}=\overline{\sigma^{(j+1)}}+\overline{r^{(j+l)}}
$$

which implies that

$$
\bar{\sigma}=\overline{\sigma^{(h)}}+\sum_{j=1}^{h} \overline{\mathbf{r}^{(j)}}
$$

Furthermore, $\sigma^{(\text {h) }}$ is a sequence such that the markings obtained along the firing of $\sigma^{(\mathrm{h})}$ are distinct markings. Similar to (6-1), we have

$$
\left|\overline{\sigma^{(h)}}\right| \leq \operatorname{card}\left(R_{c}\left(M_{0}\right)\right)=(k+1)^{|P|}
$$

Combining (6-4) and (6-5), we have

$$
0 \leq \bar{\sigma}-\mathrm{y} \leq(\mathrm{k}+1)^{|\mathrm{P}|} \cdot \mathrm{I}_{|\mathrm{P}|} \quad\left(\mathrm{I}_{|\mathrm{P}|} \text { is a }|\mathrm{P}| \times 1 \text { identity matrix }\right)
$$

with $y=\sum_{j=1}^{h} \overline{r^{(j)}}$. The fact that $y$ is also a $T$-invariant leads to the conclusion.

From the above proof we claim that we can always take $k^{\prime}$ to be $\operatorname{card}\left(R_{c}\left(M_{0}\right)\right.$ ). However a tighter bound can be obtained from the following relation

$$
k=\max _{p \in P}\left\{M_{0}(p)+\left(\left.\right|^{\bullet} p|+| p^{\bullet} \mid\right) k^{\prime}\right\}
$$

which implies:

$$
\mathrm{k}^{\prime}=\min _{\mathrm{p} \in \mathrm{P}} \frac{\mathrm{k}-\mathrm{M}_{0}(\mathrm{p})}{|\cdot \mathrm{p}|+\left|\mathrm{p}^{\bullet}\right|}
$$


Furthermore in terms of vector norm, the above result can be equally stated as follows,

RESULT VI-1 (second formulation): For a CO net G, a prefix-closed subset $L_{c}(G)$ of $L(G)$ is a set of $k$-feasible sequences for some finite $k$ if and only if there exists finite $k^{\prime}>0$ such that for any $\sigma \in L_{c}(G),\left\|\bar{\sigma}-y_{\sigma}\right\|_{\infty} \leq k^{\prime}$, for at least one $T$ invariant $y_{\sigma}$.

\section{IDENTIFICATION OF CO NET}

From Section $I V$, we know that a $C O$ net $G$ has the properties of liveness, consistency, and reversibility. Thus given a Petri net, it would be nice if we could find out whether it is a $\mathrm{CO}$ net or not. This brings to the issue of $\mathrm{CO}$ net identification.

It is not difficult to check whether a given Petri net satisfies conditions $\mathrm{H} 1-\mathrm{H} 4$ in the definition of $\mathrm{CO}$ net (definition III-1). Conditions $\mathrm{H} 1$ and $\mathrm{H} 2$ can be checked place by place. We then remove the resource places. If the resulting net contains neither sink nor source place and if it does not contain any isolated node, condition $\mathrm{H} 4$ is satisfied. Condition H3, i.e. acyclicity, can thus be checked by using any graph theory-based approach.

In order to settle the problem of identifying $\mathrm{H} 5$, let us re-examine this condition. H5 means that for each output transition $t$ of a given Petri net $G$ satisfying $\mathrm{H} 1-\mathrm{H} 4$, there exists a T-invariant $\mathrm{y}_{\mathrm{t}}$ of the acyclic Petri net $\mathrm{G}^{\prime}$ obtained by removing the resource places, such that the support of $y_{t}$ contains, apart from other non-output transitions, one and only one output transition, that is $t$. Moreover the support of such T-invariants for all the output transitions of $G^{\prime}$ covers all the transitions in $G^{\prime}$. From this re-examination, we conclude that (7-0) must hold if $\mathrm{H} 5$ is satisfied:

$$
\begin{array}{ll}
\sum_{\mathrm{t} \in \mathrm{T}_{\text {out }}[\mathrm{s}] \geq 1,} & \forall \mathrm{s} \in \mathrm{T} \\
\mathrm{C} \mathrm{y}_{\mathrm{t}}=0 & \forall \mathrm{t} \in \mathrm{T}_{\text {out }} \\
\mathrm{y}_{\mathrm{t}}[\mathrm{s}]=0 & \forall \mathrm{s} \in \mathrm{T}_{\text {out }}-\{\mathrm{t}\}
\end{array}
$$


In the following we first investigate some features of $\mathrm{H} 5$, then propose two algorithms to identify it.

\section{VII-A: SOME NECESSARY AND SOME SUFFICIENT CONDITIONS FOR H5}

We denote by $\left(t_{1}, t_{2}\right)$, when no confusion may arise, a non-oriented path joining $t_{1}$ and $t_{2}$. Such a path is said to be simple if each place belonging to this path has exactly one input and one output transition.

For example, Figure 4 shows a general situation in which a simple nonoriented path occurs. The simple non-oriented path $\left(t_{1}, t_{2}\right)$ is $t_{1} p_{1} t_{3} p_{2} t_{4} p_{3} t_{5} p_{4} t_{2}$. Directed dotted lines represent the possible interaction of $\left(t_{1}, t_{2}\right)$ with the rest of the net.

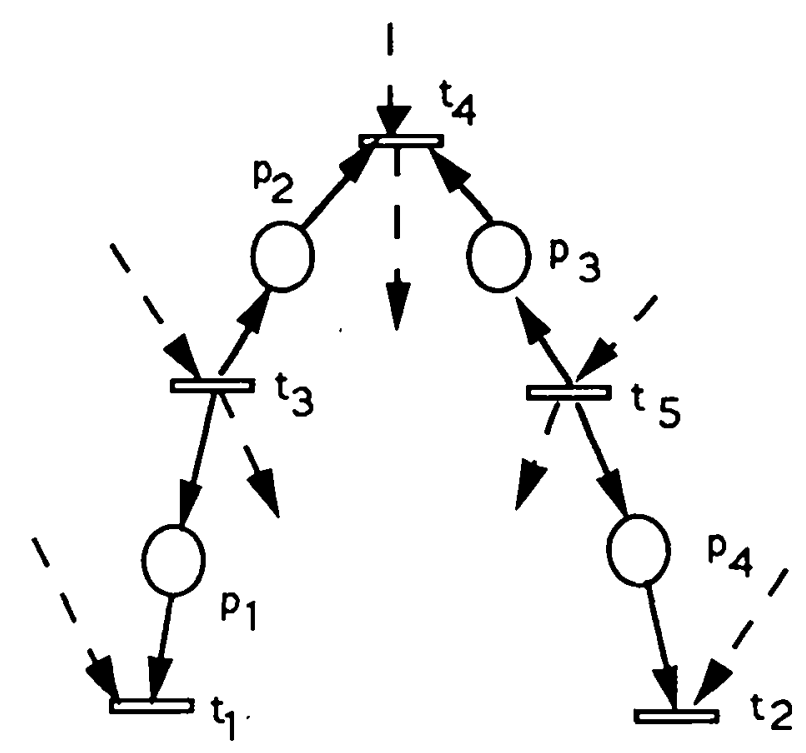

Fig. 4: A simple non-oriented path

RESULT VII-1: If a given acyclic Petri net G' satisfies H5 then there does not exist simple non-oriented path linking two output transitions.

PROOF: Consider a place $p$ with a unique input transition $t$ and a unique output transition $t^{\prime}$. It is clear that $y[t]=y\left[t^{\prime}\right]$, for any $T$-invariant $y$. As a result, for any two output transitions $t_{1}, t_{2}$ connected through a simple non-oriented path, $y\left[t_{1}\right]=y\left[t_{2}\right]$, for any $T$-invariant $y$, which implies that any $T$-invariant support containing one of the two transitions contains also the other one. This shows that H5 cannot be satisfied if there exists a simple non-oriented path connecting two output transitions.

Q.E.D. 
Remark VII-2: The insufficiency of the condition given in result VII-1 can be shown by the Petri net $G^{\prime}$ in Figure 5, which has no simple non-oriented path in it, and which does not fulfill H5 either.

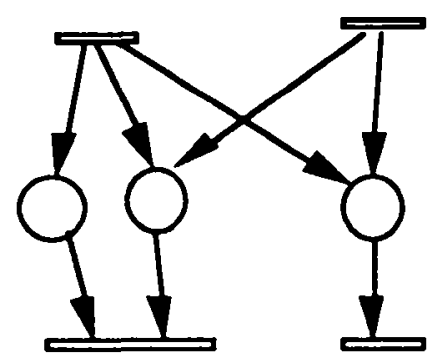

Fig. 5: A Petri net not satisfying H5 but without simple non-oriented path

We notice that the Petri net in Figure 5 is not consistent. Requiring that G' be consistent, we get the following sufficient condition.

RESULT VII-2: A given acyclic Petri net G' satisfies H5 if $\mathrm{G}^{\prime}$ is consistent, and if each minimal T-invariant support contains one and only one output transition.

PROOF: For each output transition $t$, let $y_{t}^{(1)}, y_{t}^{(2)}, \ldots, y_{t}^{\left(r_{t}\right)}$ be the set of minimal $\mathrm{T}$-invariants related to $t$. Consider the following T-invariant

$$
y_{t}=\sum_{i=1}^{r_{t}} y_{t}^{(i)}
$$

Since each minimal T-invariant support contains one and only one output transition, we have $y_{t}[s]=0, \forall s \in T_{\text {out }}-\{t\}$. Thus $\left\|y_{l}\right\| \cap T_{\text {out }}=\{t\}$. Furthermore

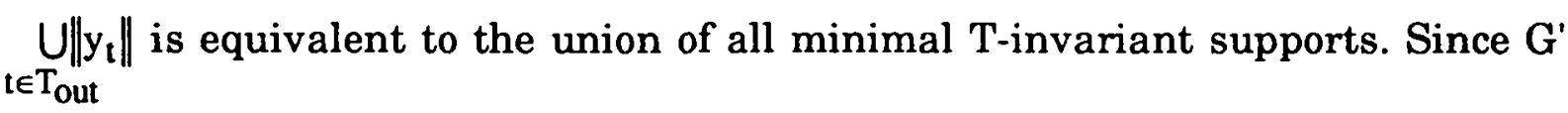

is consistent, it is equal to $T$, i.e., $\underset{t \in T_{\text {out }}}{\bigcup}\left\|y_{t}\right\|=T$.

Q.E.D.

Remark VII-3: The fact that the above condition is not necessary is shown in Figure 1(c). The incidence matrix $\mathrm{C}$ of the Petri net in Figure 1(c) is 


$$
C=\left[\begin{array}{cccccc}
1 & 0 & 1 & -1 & -1 & 0 \\
0 & 1 & 1 & -1 & 0 & -1 \\
0 & 0 & 0 & 1 & -1 & -1
\end{array}\right]
$$

Obviously this net is consistent. There are two minimal T-invariants whose supports contain $t_{5}$. They are

$$
y_{(5)}^{1}=\left[\begin{array}{llllll}
2 & 1 & 0 & 1 & 1 & 0
\end{array}\right] \text {, and } y_{(5)}^{2}=\left[\begin{array}{llllll}
1 & 0 & 1 & 1 & 1 & 0
\end{array}\right] \text {. }
$$

The two minimal T-invariants whose supports contain $t_{6}$ are

$$
y_{(6)}^{1}=\left[\begin{array}{llllll}
1 & 2 & 0 & 1 & 0 & 1
\end{array}\right] \text {, and } y_{(6)}^{2}=\left[\begin{array}{llllll}
0 & 1 & 1 & 1 & 0 & 1
\end{array}\right]
$$

while there also exists another minimal $\mathrm{T}$-invariant

$$
y_{(5,6)}=\left[\begin{array}{llllll}
0 & 0 & 3 & 2 & 1 & 1
\end{array}\right]
$$

the support of which contains both $t_{5}$ and $t_{6}$.

The sufficient characterization of $\mathrm{H} 5$ given in result VII-2 is not obvious to recognize. A sufficient characterization of $\mathrm{H} 5$ from graph-theoretical point of view can be obtained as follows .

RESULT VII-3: If a given acyclic Petri net $\mathrm{G}^{\prime}$ is consistent and the following holds:

$$
\text { H8: } \forall \mathrm{t} \in \mathrm{T},\left|\mathrm{t}^{\bullet}\right| \leq 1
$$

Then G' satisfies H5.

PROOF: From result VII-2, it is necessary to prove that each minimal Tinvariant support contains one output transition. To prove this, it is enough to show that any T-invariant support containing at least two output transitions is not a minimal support.

For this purpose, consider a T-invariant y such that $y\left[t_{1}\right]>0, y\left[t_{2}\right]>0$, for $t_{1}, t_{2} \in T_{\text {out }}$. Since $G^{\prime}$ is acyclic, it is possible to relabel the places $p_{1}, p_{2}, \ldots, p_{m}$ with $m=|P|$ such that there is no directed path from $p_{i}$ to $p_{j}$ if $i>j$.

Let us introduce a subset of transitions $\mathrm{T}^{*}=\{\mathrm{t} \in \mathrm{T} \mid \mathrm{y}[\mathrm{t}]>0\}$. We construct a $\mathrm{T}$ invariant $\mathrm{y}^{*}$ whose support is smaller than $\mathrm{T}^{*}$. This will conclude the proof. 


$$
y^{*}\left[t_{1}\right]=1 \text {, and } y^{*}[t]=0 \quad \forall t \in T_{\text {out }}-\left\{t_{1}\right\}
$$

Starting from $p_{m}$, then $p_{m-1}, \ldots, p_{1}$, we consider their input transitions and determine the value $y^{*}[t]$ as follows:

$$
y^{*}[t]= \begin{cases}0, & \text { if } t \notin T^{*} \\ \frac{1}{\left|\cdot p_{i} \cap T^{*}\right|} \sum_{s \in p_{i}^{*}} y^{*}[s], & \text { if } t \in T^{*}\end{cases}
$$

with $\mathrm{p}_{\mathrm{i}}=\mathrm{t}^{\bullet}$ which can be uniquely determined as a result of assumption H8.

Clearly for any place $p_{j}$ without output transitions belonging to $T^{*}$, we have

$$
\mathrm{y}^{*}[\mathrm{t}]=0 \quad \forall \mathrm{t} \in^{\bullet} \mathrm{p}_{\mathrm{i}} \cup \mathrm{p}_{\mathrm{i}}{ }^{\bullet}
$$

which implies

$$
\sum_{t \in p_{i}^{*}} y^{*}[t]=\sum_{t \in p_{i}^{*}} y^{*}[t]=0
$$

For any place $p_{i}$ such that at least one output transition belonging to $T^{*}$, there exists at least one input transition of $p_{i}$ belonging to $T^{*}$, i.e., $\left|\cdot p_{i} \cap T^{*}\right|>0$. From relation (7-4), we have

$$
\underset{t \in \epsilon^{\bullet}}{\sum \mathrm{p}_{\mathrm{i}} \cap \mathrm{T}^{*}}[\mathrm{t}]=\sum_{s \in \mathrm{p}_{\mathrm{i}}^{\bullet}} \mathrm{y}^{*}[\mathrm{~s}]
$$

which implies that

$$
\sum_{t \in \in_{i}^{*}} y^{*}[t]=\sum_{s \in p_{i}^{*}} y^{*}[s]
$$

as $\mathrm{y}^{*}[\mathrm{t}]=0, \quad \forall \mathrm{t} \notin \mathrm{T}^{*}$.

Relations (7-5) and (7-6) imply that $\mathrm{y}^{*}$ is also a T-invariant. Furthermore we have $\mathrm{y}^{*}\left[\mathrm{t}_{2}\right]=0$, and $\mathrm{y}^{*}[\mathrm{t}]=0 \quad \forall \mathrm{t} \notin \mathrm{T}^{*}$. Thus $\left\|\mathrm{y}^{*}\right\| \subseteq \mathrm{T}^{*}-\left\{\mathrm{t}_{2}\right\}$. 


\section{VII-B: IDENTIFICATION ALGORITHM}

When checking $\mathrm{H} 5$ for a given acyclic Petri net $\mathrm{G}^{\prime}$, we can first make use of result VII-1 to see if there exists a simple non-oriented path in G'. If not, results VII-2 and VII-3 can be further utilized to check H5. In this subsection, we propose two algorithms to tackle the identification problem. We notice that a natural way to realize (7-0) is to solve a linear programming problem as done in the following algorithm.

\section{[Algorithm VII-1]}

Input: Incidence matrix $\mathrm{C}$ of the given Petri net Output: sufficient condition for H5

Step 1: Solve the following problem (PL1)

$$
\begin{aligned}
& \min \sum_{s \in T} \sum_{t \in T_{\text {out }}} \mathrm{y}_{t}[\mathrm{~s}] \\
& \text { (PL1-1) } \\
& \text { s.t. } \sum_{t \in T_{\text {out }}} \mathrm{y}_{\mathrm{t}}[\mathrm{s}] \geq 1 \quad \forall s \in \mathrm{T} \\
& \text { (PL1-2) } \\
& C \mathrm{y}_{\mathrm{t}}=0 \quad \forall \mathrm{t} \in \mathrm{T}_{\text {out }} \\
& \text { (PL1-3) } \\
& \mathrm{y}_{\mathrm{t}}[\tau]=0 \quad \forall \tau \in \mathrm{T}_{\text {out }}-\{\mathrm{t}\}
\end{aligned}
$$

Step 2: If PL1 has a solution, then " H5 is satisfied ", otherwise, "H5 is not satisfied"

\section{[ End of Algorithm VII-1]}

Remark VII-4: In the above algorithm, the constraints of the linear programming problem PL1 are just the requirements of (7-0). The correctness of the algorithm is obvious.

Remark VII-5: We can see that using algorithm VII-1 it is straightforward to arrive at a conclusion for the identification problem. However the disadvantage that goes along with this straightforwardness is that the whole computational load is centred on one step, step 1.

In order to circumvent the drawback of algorithm VII-1, we propose the following algorithm VII-2, in which we make use of "divide and conquer" method and approach the whole problem recursively. At each iteration, we try to find out the maximal support $\mathrm{T}$-invariant concerning only one given output transition. When all such T-invariants for all the output transitions are found 
and summed up, it is sufficient to make a conclusion for the satisfiability of H5. The trick of searching the maximal support $T$-invariant at each iteration accelerates undoubtedly the speed of calculation.

\section{[Algorithm VII-2]}

Input: Incidence matrix $\mathrm{C}$ of the given Petri net

Output: satisfiability of $\mathrm{H} 5$

Declaration: $y, y_{t}$ are $|T| \times 1$ matrices

Step 1: For each $t \in T_{\text {out }}$, solve the following problem (PL2)

$$
\begin{array}{lll} 
& \max \sum_{\tau \in \mathrm{T}} \mathrm{z}_{\tau} & \\
\text { s.t. } & \mathrm{z}_{\tau} \leq \mathrm{y}_{\mathrm{t}}[\tau] & \forall \tau \in \mathrm{T} \\
& \mathrm{z}_{\tau} \leq 1 & \forall \tau \in \mathrm{T} \\
& \mathrm{C} \mathrm{y}_{\mathrm{t}}=0 & \\
& \mathrm{y}_{\mathrm{l}}[\mathrm{s}]=0 & \forall \mathrm{s} \in \mathrm{T}_{\text {out }}-\{\mathrm{t}\} \\
& \mathrm{z}_{\tau} \geq 0, \quad \mathrm{y}_{\mathrm{t}}[\tau] \geq 0 & \forall \tau \in \mathrm{T}
\end{array}
$$

Step 2: set $\mathrm{y}:=\sum_{\mathrm{t} \in \mathrm{T}_{\text {out }}} \mathrm{y}_{\mathrm{t}}$

Step 3: if $y>0$, then " H5 is satisfied ", otherwise " H5 is not satisfied " [End of Algorithm VII-2]

Remark VII-6: In the above algorithm, the functions of constraints PL2-5, and PL2-4 are respectively the same as the constraints PL1-4, and PL1-3 in algorithm VII-1. The only difference is in constraints PL2-3 and PL2-2 which manifest the fact that we try to find out, at each iteration, the maximal support T-invariant. These two constraints together with the objective function PL2-1 ensure that for each output transition $t$ the corresponding $T$-invariant obtained by solving PL2 is a maximal support T-invariant.

\section{CO NETS AND PRODUCTION MANAGEMENT}

It is interesting to recall another class of Petri nets called CFIO-decomposable Petri nets [4]. A CFIO-decomposable Petri net is a Petri net that can be decomposed into a set of consistent CFIOs. A CFIO net is defined to be a structurally conflict-free Petri net with input and output transitions but without source and sink places. The notion of CFIO-decomposable Petri net 
was used to deal with non-cyclic manufacturing system management. As such a Petri net model representing a non-cyclic manufacturing system is decomposed, if it can, into a set of consistent CFIOs, then a collection of (minimal) T-invariants whose sub-nets cover the whole net are derived from the set of CFIOs. These (minimal) T-invariants are further utilized to formulate an optimization problem whose solution gives a planning over a horizon.

Although CFIO-decomposable Petri nets are similar to CO nets, they form different Petri net classes. CFIO-decomposable Petri nets and CO nets can be reduced to the same class of Petri nets if we introduce additional constraints to both classes, namely:

(i) We define the CFIO-decomposable Petri nets as in [4], but we also require that "NC is a connected acyclic CFIO net with a unique output transition".

(ii) We consider the class of $\mathrm{CO}$ nets which can be covered by a set of $\mathrm{T}$ invariants each of which is related to a conflict-free subnet with a unique output transition.

The class of Petri nets obtained by applying these constraints is not restrictive as far as manufacturing systems are concerned. Furthermore, this class of Petri nets allows us to solve the management and the integration problems.

\section{CONCLUDING REMARKS}

This paper is part of our work on building a sound modular methodology for modelling and analysing manufacturing systems as described in the introduction. It focuses on the system integration. For this purpose we defined a class of Petri net modules called Controllable-Output nets ( CO nets) which were proved to be live, consistent and reversible. The most important result is that the integration of the $\mathrm{CO}$ net modules preserves all these properties. As a matter of fact, it was also proved that the integrated net is itself a $\mathrm{CO}$ net.

Future research is multi-fold. First the integration of decomposable Petri net modules as defined in [4] will be considered. Another important point to be addressed is to relax the constraints on the resource places. For example, resources places corresponding to buffers and transportation facilities do not satisfy assumption $\mathrm{H} 1$. 
The further step of the future research concerns the material flow coordination between the modules. This clearly involves quantitative analysis and the ultimate goal is to derive a quantitative evaluation of the integrated system.

\section{REFERENCES}

[1] T. Agerwala and Y. Choed-Amphai, "A Synthesis Rule for Concurrent Systems," Proc. 15th Design Automation Conf., Las Vegas, pp. 305-311, 1978.

[2] G.W. Brams, Réseaux de Petri: Théorie et Pratique, MASSON, 1983.

[3] G. Berthlot, "Checking Properties of Nets using Transformation," in Advances in Petri Nets 1985, G. Rozenberg (ed.), Lecture Notes in Computer Science, Springer-Verlag, pp. 19-40, 1985.

[4] G. Harhalakis, M. Leventopoulos, C.P. Lin, R. Nagi and J.M. Proth, "A class of conflict free Petri nets used for controlling manufacturing systems," Technical Report No. TR92-90, Systems Research Centre, University of Maryland, USA, 1992.

[5] I. Koh, and F. DiCesare, "Modular transformation methods for generalized Petri nets and their application to automated manufacturing systems," IEEE Trans. on systems, man, and cybernetics, vol, 21, no.6, 1512-1522, 1991.

[6] I. Koh, and F. DiCesare, "Synthesis methods using deviation bounds in automated manufacturing systems," Proc. IEEE Int. Conf. on Robotics and Automation, pp. 1025-1030, 1992

[7] B.H. Krogh and C.L. Beck, "Synthesis of Place/Transition Nets for Simulation and Control of Manufacturing Systems," Proc. IFIP Symp. Large Scale Syst., Zurich, 1986.

[8] K.H. Lee and J. Favrel, "Hierarchical Reduction Method for Analysis and Decomposition of Petri Nets," IEEE Trans. Systems, Man, and Cybernetics, vol. SMC-15, No. 2, pp.272-281, 1985. 
[9] K.H. Lee, J. Favrel, and P. Baptiste, "Generalized Petri Net Reduction Method," IEEE Trans. Systems, Man, and Cybernetics, vol. SMC-17, No. 2,pp.297-303, 1987

[10] T. Murata, "Petri nets: properties, analysis and applications," Proceedings of IEEE, vol. 77, no. 4, pp.541-580, 1989.

[11] Y. Narahari and N. Viswanadham, "A Petri Net Approach to the Modelling and Analysis of Flexible Manufacturing Systems," Annals of Operations Research, vol. 3, pp. 449-472, 1985.

[12] V.M. Savi and X.L. Xie, "Liveness and boundedness analysis for Petri nets with event graph modules," in Application and Theory of Petri Nets 1992, K. Jensen (ed.), Lecture Notes of Computer Science, Springer Verlag, pp. 328-347, 1992.

[13] M. Silva and R. Valette, "Petri nets and flexible manufacturing," in Advances in Petri Nets 1989, G. Rozenberg ed., Lecture Notes in Computer Science vol. 424, Springer Verlag, pp. 374-417, 1989.

[14] I. Suzuki and T. Murata, "A Method for Stepwise Refinement and Abstraction of Petri Nets," J. of Computer and System Sciences, Vol. 27, pp.51-76, 1983

[15] R. Valette, "Analysis of Petri Nets by Stepwise Refinements," J. of Computer and System Sciences, Vol. 18, pp.35-46, 1979

[16] M.C. Zhou, F. Dicesare, and A.A. Desrochers, "A Top-down Modular Approach to Synthesis of Petri Net Models for Manufacturing Systems," Proc. of IEEE Robotics and Automation Conf., pp. 534-539, Scottsdale, AZ, 1989 


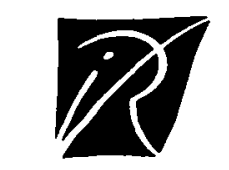

Unité de Recherche INRIA Lorraine

Technopôle de Nancy-Brabois - Campus Scientifique

615. rue du Jardin Botanique - B.P. 101 - 54602 VILLERS LES NANCY Cedex (France)

Antenne de Metz

Technopôle de Metz. 2000 - Cescom - 4, rue Marconi - 57070 METZ (France)

Uniti de Recherche INRIA Rommes IRISA. Canpus Universitaire de Beaulieu 35042 RENNES Cedex (France)

linité de Recherche INRIA Rhône-Alpes 46, avenue Félix Viallet - 3803/ GRENOBLE Cedex (France)

Unité de Rechercle INRIA Rocyuencourt Domaine de Voluceau - Rocquencourt - B.P. 105 - 78153 LE CHESNAY Cedex (France)

Unite de Recherche I: RRIA Sophia Antipolis 2004, route des Lucioles - B.P. 93 - 06902 SOPHIA A.NTIPOI.IS Cedex (France)

EDITEUR

INRIA - Domaine de Volucealu - Rocquencourt - B.P. I05 - 78153 LE CHESNAY Cedex (France)

ISSN $0249-6399$ 